\title{
La transmisión textual de la traducción de la Divina Comedia (1515): ¿del impreso al manuscrito?
}

\author{
The textual transmission of the Castilian translation of the Divine \\ Comedy (1515): From print to manuscript?
}

\author{
Cinthia M. Hamlin \\ Seminario de Edición y Crítica textual (SECRIT) \\ Universidad de Buenos Aires
}

RESUMEN: El texto de la primera traducción impresa de la Divina Comedia (Burgos, 1515) se conserva sólo en un manuscrito (HSA, B2183), considerado hasta ahora el texto de base que se usó en la imprenta. Este tema ha sido poco discutido, tal vez por la falta de un estudio minucioso que aborde el manuscrito y los problemas de su transmisión textual. En el presente trabajo, intentaremos problematizar la relación de filiación que se ha dado por sentada entre el testimonio manuscrito y el impreso, a través de un cotejo que se enfocará especialmente en los versos enmendados y en las variantes tanto léxicas como sintagmáticas.

Palabras clave: Divina Comedia, traducción, testimonio manuscrito e impreso, cotejo, filiación.

ABSTRACT: The first printed translation of the Divine Comedy (Burgos, 1515) is conserved in only one manuscript (HSA, B2183), which has hitherto been considered to be the source text used for its printing. This issue has not been the subject of much debate, possibly due to the lack of a thorough study of the manuscript and its textual transmission problems. In the present study we will try to discuss the assumed filiation between the manuscript and the printed version, through a collation of both testimonies that will focus on the manuscript's emended verses as well as on the lexical and syntagmatic variants.

Keywords: Divine Comedy, translation, manuscript and printed testimonies, collation, filiation. 
La primera traducción impresa de la Divina Comedia, que salió de las prensas de Burgos en abril de 1515, fue realizada por Pedro Fernández de Villegas, arcediano de dicha ciudad, a pedido de Doña Juana de Aragón, Duquesa de Frías y Condesa de Haro, hija natural de Fernando el Católico. Esta versión en coplas de arte mayor del Infierno está acompañada, además, de un extenso comentario del mismo traductor, que se encuentra alrededor de cada una de las coplas, envolviéndolas, bajo el mismo formato "en cebolla", que Hernán Núñez había adoptado en su Comentario de las Trescientas (1489-1505) ${ }^{1}$. Se trata éste de un texto bastante olvidado y poco estudiado por la crítica, no sólo desde el punto de vista literario o traductológico, sino también desde el punto de vista de su transmisión textual. Los escasos estudios sobre el mismo se limitan en su mayoría a analizar fragmentos de la traducción —abordando el problema de la lengua, del léxico, de sus amplificaciones u omisiones - con el fin de dar cuenta de la marcada intencionalidad didáctico-moral de Villegas ${ }^{2}$, y no le prestan demasiada atención a su funcionalidad contextual (política y/o cultural), ni a la complejidad que la glosa le otorga a la obra ${ }^{3}$.

Respecto de las problemáticas concernientes a su tradición textual, lo poco que se ha dicho se encuentra en trabajos de carácter general, es decir, en catálogos o estudios generales sobre la traducción en la Edad Media. Según el inventario que nos ofrecen Alvar y Lucía Megías (2009: 298), se tienen noticias de 45 ejemplares de este impreso, que se hallan esparcidos en diferentes bibliotecas europeas y norteamericanas. Las variaciones derivadas del agregado sucesivo, al final de la traducción, de diversos postliminares - fe de erratas, ultílogo y otros textos poéticos (ya no del cuño de Villegas)- (Morreale, 2006 [1967]: 14-5) dan cuenta de los diferentes estados textuales de estos testimonios. Sin embargo, es preciso notar que las coplas de arte mayor que nos trans-

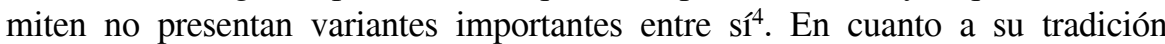

\footnotetext{
${ }^{1}$ Seguimos en la nominación de este formato a Rodríguez Velasco (2001).

${ }^{2}$ Véanse al respecto: Beltrani (1915); Arce (1965: 26-29); Fine (1981); Andreu Lucas (1995). Además de Fine, único que se centra con prioridad y exhaustivamente en este comentario aunque también con el objetivo de probar la intención didáctico-moralizante que determina la interpretación del arcediano - a través de un análisis pormenorizado de su selección de fuentes bíblicas, patrísticas, clásicas y de autores vernáculos-, Recio ha también ya señalado la necesidad de estudiar el comentario y sus relaciones con el texto poético, pero le ha dedicado a este análisis sólo unas pocas páginas (1999 y 2003: 328-30).

3 Hemos intentado subsanar estas "lagunas críticas" en nuestros últimos trabajos. Véase Hamlin (2011, 2012a, 2012b, 2012c, 2012d, 2013b, 2014).

${ }^{4}$ Las variantes significativas más comunes que se introducen en la imprenta son aquellas derivadas de problemas de conteo para completar la caja [véase: Rico (2000: 223-241)]. En el caso de un texto con comentario como el nuestro, en el cual la copla se encuentra insertada en él a modo de enclave a través de un cuadrado lo suficientemente ancho como para acoger los versos más largos, esta problemática se desplaza al comentario, espacio textual variable en su cantidad de líneas y que le permite al cajista encontrar una eventual solución.
} 
manuscrita, se conserva un único testimonio (ms. B2183 de la Hispanic Society of America) que parecería diferir del texto que nos transmiten los impresos simplemente por carecer del aparato paratextual (preliminares y postliminares) y de la extensa glosa, la cual se reemplaza con "glosillas" interlineares muy esporádicas. Según la descripción de Dutton (1982: 95) se trata de un manuscrito autógrafo, conclusión a la que llega basándose en la retiración de la tapa, según asume Faulhaber (1983: 516-8). Alvar, además, en sus últimos trabajos (1990: 34; 2009: 97 y 2010: 342) señala que seguramente es el mismo texto que se publicó por las prensas de Fradrique de Basilea en Burgos, 1515, como indicaba también Morreale (2006 [1967]: 225). Los datos otorgados por todos estos críticos han sido poco discutidos, seguramente por la falta de un estudio minucioso que aborde el manuscrito y los problemas de su transmisión textual ${ }^{5}$.

En el presente trabajo nuestro objetivo es, por tanto, problematizar la relación de filiación que se ha dado por sentada entre el texto que nos trasmite el manuscrito y el que nos transmiten los impresos, de los cuales utilizaremos el ejemplar conservado en la Real Biblioteca de Madrid, bajo la signatura I-B-21. Con este fin, cotejaremos los dos testimonios, enfocándonos especialmente en las variantes léxicas y sintagmáticas, y en los versos que aparecen enmendados en el manuscrito. Asimismo, nos será de utilidad, por un lado, cotejar estas variantes con el texto dantesco y, por el otro, con la glosa, pues algunas veces allí el traductor-comentador, mientras parafrasea el verso, ofrece nuevas variantes.

\section{PRIMER PROBLEMA DE FILIACIÓN: LAS VARIANTES}

Es preciso señalar, primero, que las diferentes lecciones obtenidas del cotejo minucioso entre el texto del manuscrito y el del impreso elegido impedirían, en principio, filiar uno y otro testimonio. Nos dedicaremos en este primer apartado a las lecciones más significativas, a saber, aquellas que resultan de variantes léxicas o sintagmáticas en el seno del verso. Para analizar este tipo de variantes tendremos en cuenta principalmente su relación de más o menos cercanía con el verso fuente en toscano y, además, el tipo de relación que se pueda establecer con la lección que transmite la glosa del impreso cada vez que Villegas parafrasea y comenta el pasaje en cuestión. Con estos fines, comenzaremos con un grupo de ejemplos que nos ayuden tal vez a establecer ciertas nociones preliminares acerca de la filiación de ambos testimonios ${ }^{6}$ :

\footnotetext{
${ }^{5}$ Es preciso aclarar que con posterioridad a la finalización y presentación de este artículo salió publicada, en noviembre de 2011, la tesis que el prof. Mondola le dedica a nuestro texto, que desde nuestro punto de vista presenta varios problemas, en especial en lo que respecta a su abordaje filológico y ecdótico. Reenvíamos a nuestra reciente reseña (Hamlin, 2012-2013).

${ }^{6}$ Citamos de acuerdo al número de canto, copla y verso, para evitar poner a cada paso el número de folio. De todos los ejemplares impresos conservados, de aquí en más utilizaremos
} 


\begin{tabular}{|c|c|c|c|}
\hline \multirow{2}{*}{1} & \multirow{2}{*}{ VII, $5 \mathrm{~h}$} & Imp. & Los unos con otros venirse a encontrar \\
\hline & & Ms. & Los unos con otros venirse a topar \\
\hline \multirow{2}{*}{2} & \multirow{2}{*}{ XIII, $16 \mathrm{~b}$} & Imp. & Mas cahe por do la fortuna la adiestra \\
\hline & & Ms. & Mas cahe por do la fortuna le muestra \\
\hline \multirow{2}{*}{3} & \multirow{2}{*}{ XXII, 21b } & Imp. & Muy presto con garfios agudos torcidos \\
\hline & & Ms. & Ligeros con garfios agudos torcidos \\
\hline \multirow{2}{*}{4} & \multirow{2}{*}{ XXXIII, $6 \mathrm{~g}$} & Imp. & Oý como abaxo sonaban clavando \\
\hline & & Ms. & Oý como abaxo clababan çerrando \\
\hline \multirow{2}{*}{5} & \multirow{2}{*}{ XIII, $17 \mathrm{e}$} & Imp. & Serán nuestras carnes aquí derrostradas \\
\hline & & Ms. & Serán nuestras carnes aquí destrozadas \\
\hline
\end{tabular}

Cuadro I, 1

En el primer caso, la comparación con el hemistiquio dantesco — "percoteansi "ncontro" (VII, 28) ${ }^{7}$, cuya traducción sería "se golpeaban al encontrarse"-, demuestra que la lección del impreso, aunque en el contexto de un verso bastante libre, resulta más cercana al toscano que la del manuscrito en cuanto que traduce conservando en castellano el lexema de la lengua fuente (incontr-). Notemos, sin embargo, que la idea del golpe parece quedar omitida en las versiones castellanas, aunque podría postularse que la variante "topar" del manuscrito incluye como rasgo semántico el "choque". Sucede algo similar en el ejemplo 3: al confrontar la traducción con el verso de la Commedia - “con tutt' $i$ raffi, e assai prestamente" (XXII, 147) — encontramos que la variante del impreso ("muy presto") intenta conservar el lexema del toscano mientras que la opción del manuscrito conserva el sentido pero con una traducción más libre. En ambos casos vemos cómo mientras que la versión del impreso está todavía muy pegada al texto fuente, la del manuscrito parece ser una versión más acabada y, en este sentido, seguramente posterior. La primera pregunta que surge, pues, es cómo se explica, de ser este manuscrito el testimonio utilizado en la imprenta, que a partir de una lección más libre respecto del toscano, se llegue en la imprenta justamente a una más literal. Lo mismo se puede plantear en casi todos los casos que analizaremos en el Cuadro I, 2 (v. $i$. ejemplos $6,7,8$ y 9).

el impreso I-B-21, de la Biblioteca del Palacio Real: Pero Fernández de Villegas (1515). En nuestra transcripción se regulariza y moderniza el uso de mayúsculas y se usan criterios actuales para la acentuación, puntuación y separación de palabras. Las cursivas serán siempre nuestras.

${ }^{7}$ Es necesario aclarar que Villegas utiliza como texto fuente el impreso de la Commedia de 1481 que contaba con el "Comento" de Landino, del cual traduce también partes del comentario en su glosa. Para facilitar la exposición, sin embargo, seguiremos el texto establecido por Petrocchi (1994) en su edición crítica el cual no presenta variantes significativas respecto del impreso en ninguno de los versos que citaremos aquí, sólo mínimas divergencias ortográficas. Una copia digital de la Commedia de 1481 puede ser consultada en <http://gallica.bnf.fr >. 
Los otros ejemplos de este cuadro (2, 4 y 5) presentan, en cambio, variantes que parecen ser igual de libres en relación con el texto dantesco. En el ejemplo 2, el toscano decía "dove fortuna la balestra" (98), es decir, donde la fortuna la "ballestea", la arroja, la expele. El verso de Villegas omite la imagen del alma siendo despedida (imagen en íntima relación con el tipo de pena: los suicidas que desdeñaron su cuerpo, son ahora desdeñados) y opta por otra visión de la fortuna utilizando el verbo "adiestrar", en el sentido de guía, que se utilizaba muchas veces en un contexto semántico cristiano ${ }^{8}$. El "mostrar" del manuscrito sería, pues, una variante de estilo, que aunque se desliga de una connotación tan cristiana, conserva el sentido primigenio que aporta Villegas con su traducción. La variante que nos trasmite el comentario - "a do la guía la fortuna" (fol. 169v) — parecería demostrar, sin embargo, que tal vez haya sido "la adiestra" la primera opción del traductor.

En el ejemplo 4, la fuente toscana trasmitía: "e io sentí chiavar l'uscio di sotto/a l'orribile torre (...)" (XXXIII, 46-7) (cuya traducción literal sería "y yo oí clavar la puerta de abajo [...]"). Villegas traduce estos versos omitiendo el objeto del chiavare — "uscio"- que pasa ahora a ser, según el verso que sigue en ambos testimonios ("la torre espantosa que nos encerraba" 6h), la torre. Para que le dé el cómputo silábico, agrega un término: en el impreso "sonaban" que acentúa el aspecto sensitivo de la acción y en el manuscrito "cerrando", que esclarece la acción aludida con el "chiavare". Como sucede muchas otras veces cuando hay una omisión, en la glosa repone el objeto omitido: "oxó como enclavaban abaxo la puerta" (fol. 306v). Las variantes parecen ser, pues, opciones de estilo, siendo la del manuscrito (clavaban cerrando la torre) una opción tal vez más natural que la del impreso (sonaban clavando la torre) en cuanto que la colocación clavar + torre es en castellano una combinación léxica menos artificiosa que sonar + torre.

En el último caso el texto dantesco decía "qui le strascineremo (...)" (XIII, 106), cuya traducción literal sería "aquí los arrastraremos". El referente del pronombre "le" lo encontramos unos versos antes: "nostre spoglie" (XIII, 103) (nuestros despojos). Villegas traduce mediante una modulación, recurso que se subdivide en varios tipos, en este caso "visión activa por pasiva", pues repone el referente del pronombre objetivo y lo convierte en sujeto gramatical paciente ("serán nuestras carnes aquí derrostradas/destrozadas") 9 . En el comentario, el

\footnotetext{
${ }^{8}$ El sintagma "Dios que os adiestra", por ejemplo, aparece varias veces en el Cancionero de Marcuello (Blecua, 1987: 51 y 53) y "[s]y Dios me adiestre", a su vez, en el Cancionero de Montoro (Ciceri, 1991: 317) y en el Cancionero castellano de París (Maguire, 1995: fol. 94r). Véanse más ejemplos de este uso en el CORDE, por medio de la consulta "adiestra" y "adiestre" entre las fechas 1200 y 1500.

${ }^{9}$ Para los procedimientos técnicos de traducción y sus clasificaciones seguimos a Vázquez-Ayora (1977). Los distintos tipos de modulación se encuentran clasificados en las páginas 293-313.
} 
arcediano introduce el mismo verso del impreso pero agrega algo más: "serán nuestras carnes aquí derrostradas y arrastradas los rostros por el suelo" (fol. 170r). Siendo que una versión más literal del "strascineremo" - "serán arrastradas" - habría sido posible en cuanto a que el cómputo silábico y la rima se mantienen —el acento del "aquî" impediría la sinalefa—, el "derrostradas" parece ser una opción del traductor para inducir un patetismo mayor, opción confirmada por la glosa, donde incluye la opción más literal "arrastrar", pero en relación a la imagen del rostro arrancado de la carne. En este sentido, el "destrozadas" parece ser una opción más lejana respecto del texto fuente, y se podría postular, teniendo en cuenta la rareza del verbo "derrostrar", que podría ser una lectio facilior, error en el que se incurre al copiar el testimonio. Podría ser también una variante de estilo, en la que se allana el texto, aunque en ese caso no se explicaría cómo no se eligió el término "arrastrada", más cercano al del toscano. De una u otra manera, sería ésta una prueba más para probar nuestra hipótesis de que la versión del manuscrito sería posterior.

La relación de las variantes con la lección que nos trasmite la glosa será más reveladora en los siguientes ejemplos:

\begin{tabular}{|c|c|c|c|}
\hline \multirow{2}{*}{6} & \multirow{2}{*}{$\mathrm{V}, 11 \mathrm{~g}$} & Imp. & Y vimos después a aquel grande Archiles \\
\hline & & Ms. & Y vimos después a aquel pérfido Archiles \\
\hline \multirow{2}{*}{7} & \multirow{2}{*}{ IX, $2 \mathrm{~g}$} & Imp. & Que siendo troncadas gran miedo me han dado \\
\hline & & Ms. & Que siendo troncadas me ponen cuidado \\
\hline \multirow{2}{*}{8} & \multirow{2}{*}{ XVIII, $18 \mathrm{~d}$} & Imp. & Gridó contra mi por qué me has reguardado \\
\hline & & Ms. & Gridó contra mi por qué me has remjrado \\
\hline \multirow{2}{*}{9} & \multirow{2}{*}{ XXIX, 6b } & Imp. & De aquesto fablando hasta el otro valle \\
\hline & & Ms. & De aquesto tratando hasta el otro valle \\
\hline \multirow{2}{*}{10} & \multirow{2}{*}{ XXXI,5b } & Imp. & Te digo primero que mas te adelantes \\
\hline & & Ms. & Te digo y sabrás sin que mas te adelantes \\
\hline
\end{tabular}

Cuadro I, 2

En el primer caso, la lección del impreso se atiene más al texto dantesco, que decía "E vedi 'l grande Achille" $(\mathrm{V}, 65)$ mientras que el manuscrito introduce con el nuevo adjetivo un juicio de valor moralizante, acorde con la tendencia general de la traducción. Resulta interesante, sin embargo, que en el comentario del impreso cuando retoma este verso dice "y bimos después a aquel pérfido Archiles" (fol. 81r). Así, la glosa del impreso revela que la relación entre los dos testimonios es compleja. Un caso bastante similar a éste es el del ejemplo 7: encontramos aquí nuevamente que la lección del impreso es una versión más cercana al texto fuente, que rezaba "ma non di men paura il suo dir dienne/perch' io traeva la parola tronca" (IX, 13-4). Aunque en el impreso 
traduce el sintagma mediante una modulación (lo contrario negativado), mantiene una traducción literal del término "paura" (miedo), término que en el manuscrito se traduce mediante la locución verbal "poner cuidado", más alejada del término dantesco por cuanto resalta la consecuencia del sentimiento $-\mathrm{y}$ por tanto el accionar-, y no el sentimiento en sí. En la glosa del impreso dice, sin embargo: "pues seyendo estas palabras troncadas (...) como suelen hacer los cuidosos y pensatibos (...) dize el Dante ponen me en cuidado que lo malo dellas (...) se nos diga" (fol. $126 \mathrm{v}$ ). Se deduce de estos dos casos, por un lado, que la versión del manuscrito es posterior y que, en estos casos, modifica la primera versión más apegada al toscano según una tendencia más moralista (aún) y, por otro, que la glosa parece confeccionarse siguiendo una versión similar (o la misma) a la del manuscrito, pues presenta lecciones similares.

Los otros ejemplos poseen características análogas. El segundo (ej. 8) se presenta como otro caso de la tendencia del impreso a conservar lexemas similares, en esta ocasión, por medio de un calco de "riguardar". El verso dantesco completo decía "[perché se' tu sí gordo/] di riguardar piú me che li altri brutti" (XVIII, 118-9). La opción del manuscrito "remirado" parece ser nuevamente una corrección de estilo, que se deshace del calco por medio de una opción más natural. En la glosa del impreso, sin embargo, dice: "por qué me has remirado más que a los otros" (fol. 208v). Sucederá algo similar en el ejemplo 9, donde la lección del impreso "fablando" es otra vez una traducción más literal del verso dantesco _-"cosí parlammo infino al loco primo"(XIXX, 37)_ que la que nos trasmite el manuscrito ("tratando"), que en este caso no parece implicar ningún cambio importante, más que ser otra opción de estilo. La glosa, sin embargo, trasmite esta misma variante: "tratando de esto que ha dicho fasta el otro valle" (293v). En el último caso (ej. 10), el toscano decía " $e$ disse: pria che noi siam piú avanti" (XXXI, 29). "Primero" es pues un calco de "pria" (forma poética de "prima"), que en italiano significa "antes de". La versión del manuscrito parece corregir este calco mediante una transposición (cambio de categoría o estructura gramatical, [Vázquez-Ayora, 1977: 268-274]) que genera a su vez una modulación: introduce un verbo que duplica la función de la didascalia "(te digo y sabrás"), seguido de la preposición "sin". Por tanto, en "te digo sin que te adelantes" se mantiene el significado del "antes que" aunque cambiando la perspectiva temporal por una de privación o carencia. Luego glosa: "asý que le dize antes que te adelantes ni vayas mas adelante te fago saber que non son torres las que veys" (293r). Una vez más, la variante que presenta la glosa, igual a la del manuscrito, podría indicar que el testimonio utilizado para confeccionar el comentario traía ya esta misma variante.

El panorama se torna aún más complejo al estudiar los siguientes ejemplos en su relación con la glosa: 


\begin{tabular}{|c|c|c|c|}
\hline \multirow{2}{*}{11} & \multirow{2}{*}{ IX, $7 \mathrm{~g}$} & Imp. & Y díxome guarda si quieres mirar \\
\hline & & Ms. & Y díxome vuelve si quieres mirar \\
\hline \multirow{2}{*}{12} & \multirow{2}{*}{ XXXIV, 6d } & Imp. & Y todo fablar es en esto cortado \\
\hline & & Ms. & Y todo fablar es en esto abreviado \\
\hline \multirow{2}{*}{13} & \multirow{2}{*}{ XXVI, $14 \mathrm{~g}$} & Imp. & La nuestra vejez y pereza peor \\
\hline & & Ms. & La nuestra vejez y tardana pereza peor \\
\hline
\end{tabular}

Cuadro I, 3

En el primer caso vemos nuevamente que la versión del impreso trata de conservar el lexema — pues el toscano decía: "guarda, mi disse, le feroce encine" (IX, 45) - aunque utilizándolo, esta vez, según el significado castellano, como da cuenta la glosa: para se guardar dellas si quiere mirar y conocer las furias feroces" $(129 \mathrm{v})$. Igualmente, repone mediante otro verbo - mirarel sentido principal del guardare italiano. El manuscrito opta, sin embargo, por agregar en el discurso de Virgilio un verbo de movimiento ausente en el texto fuente. Lo interesante es que en la glosa nos dirá: “(...) a las quales dize Virgilio que se buelua a mirar y las conozca; para se guardar dellas si quiere mirar y conocer las furias feroces de aquellas moradas" (129v.). Parece, pues, tener las dos versiones en cuenta en este comentario. En el último caso, traducción del "però ch'ogni parlar sarebbe poco" (XXXIV, 24), se puede observar también una variante de estilo (cortado/abreviado), ninguna más cercana al término dantesco que la otra. En la glosa, Villegas comenta: "todo fablar verná corto y breve" (fol. 313v). Una vez más, el comentario presenta una versión en la que se dan de la mano las dos variantes.

El último caso, presenta una enmienda al margen que es fruto de lo que sería una segunda mano, diferente de la del copista/traductor y bastante posterior, que corrige la variante siguiendo el impreso. La lección primigenia del manuscrito (tardanza) parece ser, entonces, una excepción de la tendencia ya señalada: en este caso es el manuscrito el que conserva el lexema del toscano, que rezaba: "io e' compagni eravam vecchi e tardi" (XXVI, 106). Sin embargo "tardo", en su primera acepción según el Vocabolario etimologico della lingua italiana (Pianigiani, 1993: 1408), significa "pigro" (perezoso) y en la edición de la Divina Commedia de Nicola Fosca (2003), al anotar el verso 106 parafrasea "vecchi e stanche" (viejos y cansados). Pereza, por tanto, es la opción semánticamente más cercana al término dantesco. En este caso, en la glosa comenta: "que ya los empachaba la vejez y la tardanza y la pereza que es peor que la vejez" (265v). Aunque se incluyen aquí ambas lecciones, por el orden sintagmático es evidente que la proposición relativa "que es peor que la vejez" estaría modificando a "pereza" y, por tanto, dando cuenta de la lección "y pere$z a$ peor" del impreso. Por tanto, el cotejo de todas estas lecciones con la glosa parecería revelar una indecisión del traductor a la hora de elegir la mejor op- 
ción, que encuentra salida en la glosa donde, sin la restricción de la métrica y amparándose en la técnica de la amplificatio típica de la enarratio poetarum, puede soltar la pluma e incluir las dos variantes.

Para ir aproximándonos a una conclusión, señalemos que el análisis de las variantes léxicas y estructurales nos ha permitido observar, por un lado, que el testimonio manuscrito presenta lecciones que son fruto de una traducción más libre, lo cual, a su vez, nos permitiría descartar la hipótesis de una filiación entre el texto del manuscrito y el del impreso, ya que de una lección más alejada del toscano difícilmente se llegue en la imprenta a una tan literal. Por el otro, hemos aportado muchos otros ejemplos que prueban que el manuscrito sería una versión posterior de la traducción, que corrige por cuestiones ya sean de estilo (siendo las más comunes la supresión de los calcos y la naturalidad en la lengua meta) ya sean morales la versión primera, cuyo testimonio más cercano es el del impreso. Por último, el análisis de las variantes que nos trasmite la glosa nos ha sugerido que tal vez el testimonio manuscrito $-\mathrm{O}$ mejor dicho, uno similar y anterior - haya sido utilizado o al menos consultado para su confección. Intentaremos corroborar y profundizar algunas de estas conclusiones en el análisis del segundo tipo de variantes: las enmiendas.

\section{SEGUNDO PROBLEMA DE FILIACIÓN: LAS ENMIENDAS}

Las variantes entre el manuscrito y el impreso que resultan tal vez más evidentes a primera vista son aquellas fruto de una tachadura en el primero. En este sentido, este tipo de enmienda funciona de dos maneras opuestas: 1) omitiendo un término presente en la versión del impreso (cuadro II, 1); 2) omitiendo un término ausente (cuadro II, 2). De esta manera, el primer caso da como resultado una lección diferente a la del impreso (por carecer de algún término) y en el segundo caso, una igual. Veamos unos ejemplos:

\begin{tabular}{|c|l|ll|}
\hline \multirow{3}{*}{1} & \multirow{2}{*}{ III, 9c } & Imp. & Pues no lo menciones mas pasa y procura \\
\cline { 3 - 4 } 2 & \multirow{2}{*}{$\mathrm{XV}, 7 \mathrm{~g}$} & Ms. & Putes no lo menciones mas pasa y procura \\
\cline { 3 - 4 } & & Ms. & Que va come veys con tan poca alegría \\
\hline
\end{tabular}

Cuadro II, 1

\begin{tabular}{|c|l|ll|}
\hline \multirow{3}{*}{3} & \multirow{2}{*}{ XIII, 23e } & Imp. & Y aun sino fuese porque alguna vista \\
\cline { 2 - 4 } 4 & \multirow{2}{*}{ XXI,15c } & Ms. & Y aun \\
\cline { 2 - 4 } & & Ms. & Asý los vino fuese porque alguna vista \\
\cline { 3 - 4 } & & Asý los vi yo estar temerosos \\
\hline
\end{tabular}

Cuadro II, 2 
Señalemos, igualmente, que se encuentran en todo el manuscrito más casos del primer tipo de tachadura que del segundo (en el canto II, por ejemplo, tenemos 12 del primero y 4 del segundo). La pregunta que se deduce de estos casos es: de ser el manuscrito el original usado para la imprenta, ¿por qué se respetaron algunas enmiendas y otras (la gran mayoría) no? Una posible respuesta sería postular que estas correcciones sean posteriores, en cuyo caso tampoco se explicaría que no se haya incluido en el impreso la lección previa a la tachadura (como "más" o "muy" de nuestro cuadro II, 2). Además, esta hipótesis de una segunda corrección quedaría descartada de antemano pues en todos estos casos estas enmiendas presentan la misma tinta y la misma pesantez que el verso escrito.

En el manuscrito se encuentran, no obstante, enmiendas que sí parecerían ser fruto de una segunda corrección, con diferente letra, tinta más clara, y de menor pesantez. Este es el caso, sólo para mencionar alguno, del ej. 13 del apartado anterior. Son estas correcciones de una letra humanística y, según consideramos, se trata de la misma mano que aparece en el fol. $* 9 \mathrm{v}^{10}$ y que copia, de manera bastante desprolija, las primeras tres estrofas del segundo canto del Paraíso y la Pregunta y Respuesta de Diego de Burgos que se encuentra al final del manuscrito. No analizaremos aquí esta clase de enmienda pues, al tratarse de una mano posterior, no otorga datos funcionales a nuestro objetivo.

El tercer tipo de enmienda que presenta el manuscrito es aquella en la que se tacha la lección primigenia y se corrige por encima, esta vez sí con la misma tinta y letra que la del verso. Veremos sólo algunos casos (ver Cuadro II,3).

Varias cuestiones pueden deducirse de estos ejemplos. En primer lugar, lo más evidente es que encontramos aquí también dos tipos de enmiendas opuestas: una que, presentando por debajo la misma lección que el impreso, genera una diferente, y otra que frente a una lección diferente, genera una igual. Surge la misma pregunta, pues, que en los ejemplos analizados arriba: de ser la versión usada en la imprenta, ¿por qué se respetan unas tachaduras y otras no? Si las enmiendas son posteriores, ¿por qué no encontramos el verso original tal como está en el manuscrito por debajo de la tachadura (ej. 10, 11 y 12)?

En segundo lugar, resulta útil también aquí cotejar las variantes con el texto fuente. Recordemos primero que Villegas al traducir las tercinas en coplas de arte mayor agrega varios hemistiquios y hasta versos enteros de su propia mano, que unas veces aclaran las imágenes dantescas, volviéndolas más explícitas, otras

\footnotetext{
${ }^{10} \mathrm{La}$ introducción del manuscrito (fol. * $* \mathrm{r}-* 9 \mathrm{v}$ ) presenta un pequeño resumen de cada canto. En el folio 9v, sin embargo, la mano cambia a una letra humanística, como señala también Faulhaber (1983: pág. 517), bastante desprolija. Esta mano completa el resumen del canto XXXIII y agrega el del XXXIV. En el folio siguiente (1r) comienza el texto traducido. Es necesario aclarar que utilizamos para el ms. la foliación de Faulhaber, que numera aparte al prefacio y el resumen de los cantos utilizando un asterisco, y comienza el conteo nuevamente con 1r al comenzar la traducción.
} 


\begin{tabular}{|c|c|c|c|}
\hline \multirow{2}{*}{5} & \multirow{2}{*}{$\mathrm{X}, 12 \mathrm{e}$} & Imp. & Sy el arte que dizes no fue bien usada \\
\hline & & Ms. & Sy el arte que dizes no fue bien thata se guardaba \\
\hline \multirow{2}{*}{6} & \multirow{2}{*}{$\mathrm{X}, 12 \mathrm{~h}$} & Imp. & de pena tan cruda qual te es demostrada \\
\hline & & Ms. & de pena tan cruda quat te nunca se acaba \\
\hline \multirow{2}{*}{7} & \multirow{2}{*}{$\mathrm{X}, 18 \mathrm{~b}$} & Imp. & Lo fizo la dubda en que atento pensaba \\
\hline & & Ms. & Lo fizo la dubda en que atento pensaba mi mente pensada \\
\hline \multirow{2}{*}{8} & \multirow{2}{*}{$\mathrm{XX}, 10 \mathrm{e}$} & $\operatorname{Imp}$ & Al pie de los Alpes está aquel tendido \\
\hline & & Ms. & Al pie de los Alpes aquet se muestra tendido \\
\hline \multirow{2}{*}{9} & \multirow{2}{*}{ XXI, 19a } & Imp. & Calcabrina avante te tira y Alquino \\
\hline & & Ms. & Calcabrina te tira pasa adelante y Alquino \\
\hline \multirow[b]{2}{*}{10} & \multirow[b]{2}{*}{ XVIII,18b } & Imp. & Del fétido lodo de aquel fondo ciego \\
\hline & & Ms. & 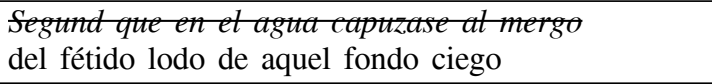 \\
\hline \multirow[b]{2}{*}{11} & \multirow[b]{2}{*}{ XXVII, $15 \mathrm{~h}$} & Imp. & Do mi antepasado no tuvo afición \\
\hline & & Ms. & $\begin{array}{l}\text { Que al mi antepasado en desteño le son } \\
\text { do mi antepasado no tuvo afición }\end{array}$ \\
\hline \multirow[b]{2}{*}{12} & \multirow[b]{2}{*}{ XXVIII,3h } & Imp. & Que con sus llagas se nos representa \\
\hline & & Ms. & $\begin{array}{l}\text { Pensarto la sangre y el alma atomenta } \\
\text { Que con sus llagas se nos representa }\end{array}$ \\
\hline
\end{tabular}

Cuadro II, 3

funcionan como comentarios morales, o que inducen a un mayor patetismo. En este sentido, los versos de los ejemplos 6, 10 y 12 son todos agregados por Villegas, así como el segundo hemistiquio del ejemplo 8. Se puede observar, pues, que las variantes más importantes se introducen justamente en los versos que genera el traductor, donde a veces se corrige incluso todo el verso.

En el verso del ejemplo 5, traducción del dantesco "elli han quell'arte, disse, male appresa" (X,77), Villegas utiliza nuevamente una modulación, en este caso, "lo contrario negativado" — "no bien" por "male" — y, luego, "lo particular por lo general" al cambiar el verbo "appresa" (aprendido) por el más general "usado". La enmienda "guardado" no produce, pues, ni un cambio importante en el sentido del verso castellano ni una aproximación mayor al sentido del texto fuente. Es, por tanto, una variante de estilo. Sucede algo similar en los versos del ejemplo 9: la lección del impreso ("avante te tira") parece una versión casi literal del toscano ("trati avanti Alichino, e Calcabrina", XX, 118) donde se mantiene, incluso, la aliteración dantesca de la oclusiva dental. En el manuscrito, sin embargo, se la enmienda para generar un verso más natural ("pasa adelante"). En el caso del ejemplo 8, encontramos también una corrección de estilo, esta vez en un hemistiquio agregado. Resulta interesante también el caso del ejemplo 11, donde las dos lecciones se atienen al sentido del texto 
fuente ("[... però sono due le chiavi/] che'l mio antecessor non ebbe care", XXVII, 105), siendo la primera versión que presenta el manuscrito otra modulación (contrario negativado) que se corrige por la misma lección del impreso, versión más literal respecto del verso dantesco.

Sin embargo, hay que notar que los versos de los ejemplos 5 y 6 pertenecen a la misma copla, el sexto y octavo verso respectivamente. Si confrontamos las variantes que presentan, advertimos que de la rima en -ada de la lección primigenia, en el manuscrito se pasa, luego de la enmienda, a una rima en -aba. Al volver a la copla, notamos una particularidad: ésta seguía la extraña estructura ABBACDDC. La corrección, por tanto, permite reincorporar la copla al esquema de rima típico del arte mayor ABBAACCA ${ }^{11}$. Estos casos estarían demostrando, por tanto, la existencia de una instancia posterior de corrección, en el que el copista/traductor se percata de la divergencia y decide enmendar.

Un caso similar es el del ejemplo 7, traducción bastante literal del v.113 de la Commedia pero que presenta un problema: el de la rima. Siendo éste el segundo verso de la copla, debería rimar con el que sigue inmediatamente y, no obstante, en el verso siguiente se lee "qual me fue agora por vos declarada"(X, 18c). Tanto la versión del impreso como la primigenia del manuscrito presentarían, por tanto, una lección errónea, que en el manuscrito la misma mano que copia el verso se encarga de enmendar ("en mi mente pensada") para conservar la rima en -ada.

Del análisis de estos casos se puede deducir, entonces, que esta versión no es la usada en la imprenta, pues estas correcciones - ya no de estilo, sino de composición del verso- deberían aparecer enmendadas en el impreso. Aclaremos nuevamente que la letra es la misma y la tinta presenta la misma intensidad y pesantez en ambos casos. La corrección parece haber sido casi inmediata. Tengamos en cuenta, además, que algunos ejemplares del impreso cuentan al final de la obra con una 'fe de erratas'12 del propio Villegas donde corrige

\footnotetext{
${ }^{11}$ Habría que aclarar en este punto que la traducción de los 34 cantos cuenta con un total de 722 coplas, de las cuales 720 siguen el esquema de rima típico. Además de la que nos concierne, sólo una copla se desvía, la primera del canto III, siguiendo, sin embargo, el mismo modelo que presenta la primera copla del Laberinto de la Fortuna (ABABBCCB) en la cual Mena honra a Juan II. El cambio del esquema de rima en este caso parece también responder a un objetivo estilístico particular: sus versos nos transmiten la traducción de la famosa inscripción de las puertas del Infierno (III, I-9), con las que comienza el viaje dantesco propiamente dicho. El objetivo es el mismo que el de Juan de Mena: aislar esta copla del resto, sea para llamar la atención sobre la gravedad de la materia que comenzará a partir de aquí, sea porque, como sabemos, se alude en estos versos a la Santísima Trinidad. La copla 12 del canto X, no obstante, no posee ninguna particularidad en su contenido que la destaque de otras tal vez más importantes y que haga pensar en una intención estilística a la hora de correrse del modelo de rima esperado.

12 Nótese que utilizamos aquí el sintagma 'fe de erratas' no según lo que éste significaba para la legislación de impresos del siglo XV-XVI, sino como lo entendemos hoy, es decir, como lista de
} 
cuestiones de ortografía, de concordancia verbal o de artículos y preposiciones erróneas. Citemos sólo dos ejemplos: "en el canto segundo en la copla que comiença de angélica voz dize vos, esta la .s. por la .z." y más adelante respecto del canto XIX corrige "en el verso tercero dize mi propio deseo tu saber mejor. Ha de dezir sabes mejor" (fol. 320v). La ausencia en esta 'fe de erratas' de una corrección tan importante como la de la rima o de la estructura de rima nos indicaría que sólo se ha percatado del error en una instancia posterior, de la que estaría dando cuenta el manuscrito donde, como ya dijimos, aparece primero la lección errónea, la cual se corrige inmediatamente.

Para ir aproximándonos a una conclusión, antes es importante mencionar que en el manuscrito encontramos varias pruebas que confirmarían el hecho de que nos hallamos frente a una copia, y no la primera versión de la traducción: por un lado, versos duplicados de los cuales se tacha el primero (fol. 87r), términos y sintagmas duplicados de los cuales se tacha el segundo (ejs. en fols. 18v y 41v), estrofas olvidadas que se agregan con la misma letra, en los márgenes inferior y superior (fols. 106v y 107r), versos salteados que se intercalan luego en letra pequeña (fols. $24 \mathrm{r}, 77 \mathrm{v}$ y $120 \mathrm{v}$ ). Ya sea por el tipo de estrofa (en el que se encadenan dos tercinas dantescas y, a veces, media más) o de verso (de rima encadenada) resulta poco probable, por no decir imposible, que estos errores se hayan producido en el proceso de traducción.

Todo lo expuesto hasta aquí nos permite corroborar, pues, que la versión que nos trasmite el manuscrito es copia de una anterior, tanto al manuscrito como al impreso, que presenta lecciones en su mayoría similares a las del impreso y otras no tanto - ej. 10, 11 y $12-$ y que se va corrigiendo a medida que se la copia (sea por cuestiones métricas o de estilo) generando un texto diferente. Las variantes del texto impreso se podrían explicar, pues, postulando que el manuscrito usado de base en la imprenta $(\alpha)$, copia de un arquetipo $[\mathrm{X}]$, presentaría ya algunas de las mismas correcciones de nuestro manuscrito y otras no. El testimonio manuscrito, en cambio, se copió con certeza de otro (subarquetipo $\beta$ ) que es el que corregía la versión más literal de $[\mathrm{X}]$ y trasmitía otras veces versos enteros diferentes. El copista-traductor transcribe las nuevas lecciones pero, en algunos casos, se encarga inmediatamente de corregirlas. Asimismo, el análisis de las variantes que nos trasmite la glosa nos ha sugerido que tal vez un testimonio similar al manuscrito haya sido utilizado o al menos consultado para su confección. En este sentido, sería otra hipótesis viable plantear que es la glosa del impreso, con sus paráfrasis y sinónimos, la que ha in-

\footnotetext{
los errores que el mismo autor observa en su libro, e inserta en él al final o al comienzo, con la enmienda que de cada una debe hacerse. En el caso del impreso I-B-21, por el tipo de enmiendas señaladas así como por su redacción es muy probable que esta "fe de erratas" sea del propio Fernández de Villegas y no del impresor o del corrector de la imprenta.
} 
fluido en las variantes del manuscrito, aunque la literalidad del comentario respecto de éstas nos hace pensar que es más probable que el movimiento de contaminación haya sido el inverso. Plantear esta última hipótesis sería suponer, pues, que el copista trabajaba a partir de una versión manuscrita que tendría las variantes analizadas pero luego corregía según el impreso y su glosa, cuestión poco probable - pues por qué no trabajar directamente copiando la versión del impreso- y desde ya muy trabajosa.

Veamos ahora unas últimas cuestiones. En un trabajo anterior (Hamlin, 2013a), luego de analizar los datos históricos que nos otorga la glosa así como el contexto histórico y cultural de la traducción, hemos replanteado el terminus a quo y el ad quem de la misma al postular que la "primera etapa hermenéutica" de esta obra, la de la traducción propiamente dicha, se llevó a cabo entre 1502 y 1510. La glosa que presenta el impreso correspondería a lo que hemos denominado una "segunda etapa hermenéutica", que se llevó a cabo entre 1510 y 1515, siendo la mayor parte seguramente posterior a 1512. Es interesante en este sentido señalar que según la descripción de Faulhaber (1983: 517), basada en este punto en Briquet (1985: 1358-62), una de las filigranas presentes en nuestro manuscrito (una víbora) se usaba entre 1510-1524. Por tanto, los aspectos codicológicos también probarían que es ésta una versión posterior a la que se realizó entre 1502 y 1510, cuyo testimonio más cercano es el del impreso de 1515. Si tenemos en cuenta que la "segunda etapa hermenéutica" se ubica entre 1510 y 1515 , no se descarta ninguna de nuestras dos hipótesis ya mencionadas: 1) un subarquetipo $(\beta)$ del testimonio manuscrito puede haberse usado en la confección de la glosa - y por tanto haberse copiado antes de 1515- pero el ms. B2183 es necesariamente posterior a la edición impresa, lo que se prueba con la cuestión de la 'fe de erratas' o 2) puede haber sido la glosa del impreso la que influyó en la versión manuscrita — caso en el cual también habría que postular la existencia de un subarquetipo b que es el que corrige la versión más literal de $[\mathrm{X}]$ y hasta trasmite versos enteros diferentes-. Sea cual fuere el caso, lo cierto es que habría que ubicar a nuestro testimonio de la Hispanic Society entre 1515 y 1524 . Teniendo en cuenta todo lo anterior, podríamos diagramar el stemma de la siguiente manera, intentando dar cuenta de la relación diacrónica y de la posible contaminación:

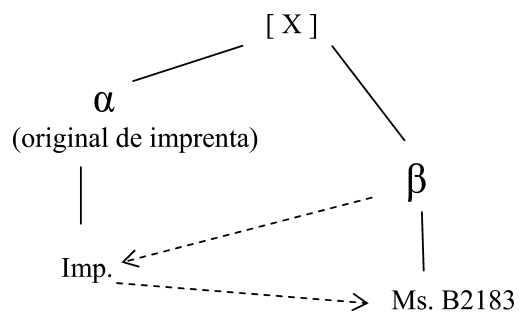

REVISTA DE FILOLOGÍA ESPAÑOLA (RFE), XCIII, 2. ${ }^{\circ}$, julio-diciembre, 2013, pp. 273-289 ISSN 0210-9174, eISSN 1988-8538, doi: 10.3989/rfe.2013.09 
Por último, no está demás mencionar dos cuestiones importantes. Por un lado, que a la hora de descartar que el texto del manuscrito B2183 sea el mismo que se utilizó en la imprenta baste tan solo traer a colación las características particulares que poseen los originales de imprenta: marcas que dejan las intervenciones legales —además de las anotaciones marginales, enmiendas, tachaduras y censuras, la rúbrica del secretario del Consejo en todas las planas- y marcas que dejan las intervenciones del impresor en el conteo del original - una serie de rayas y signaturas que delimitan las planas resultantes_- ${ }^{13}$. Todas estas características 'físicas' del original de imprenta están ausentes en nuestro manuscrito. Por el otro, que en el manuscrito, luego del Infierno, se traduce el primer canto del Purgatorio, y luego el primero del Paraíso, con la misma tinta y letra. En el folio siguiente, con una letra humanista muy desprolija se comienza con el 2 do del Purgatorio, del cual se traducen sólo 3 coplas. Todo indicaría, pues, que el texto de la Comedia que nos transmite el manuscrito se trata de una copia de un subarquetipo anterior y que, a su vez, correspondería a una versión corregida, tal vez una segunda fase dentro del proceso de traducción, posterior a la del texto que nos transmite el impreso, en la cual se intenta incluso completar toda la obra.

\section{BIBLIOGRAFÍA}

Alvar, Carlos (1990): "Notas para el estudio de las traducciones italianas en Castilla durante el siglo XV", Anuario Medieval, 2, pp. 23-41.

Alvar, Carlos (2010): Traducciones y traductores. Materiales para una historia de la traducción en Castilla durante la Edad Media, Alcalá de Henares, Centro de Estudios Cervantinos.

Alvar, Carlos y José Manuel Lucía Megías (2009): Repertorio de traductores del siglo XV, Madrid, Ollero y Ramos.

Andreu Lucas, Maribel (1995): "La amplificación en el Infierno de Dante traducido por Pedro Fernández de Villegas (Burgos 1515)", tesis doctoral inédita, Universidad de Barcelona.

Arce, Joaquín (1965): "La lengua de Dante en la Divina Comedia y en sus traductores españoles", Revista de la Universidad de Madrid, 14, pp. 9-48.

Beltrani, Armida (1915): "D. Pedro Fernández de Villegas e la sua traduzione della prima cantica della Divina Commedia”, Giornale Dantesco, 23, pp. 254-293.

Blecua, José Manuel (ed.) (1987): Pedro Marcuello, Cancionero, Zaragoza, Institución Fernando el Católico.

Briquet, Charles-Moïse (1985): Les Filigranes. Dictionnaire historique des marques du papier dès leur apparition vers 1282 jusqu'en 1600, New York, Hacker Art Books. <http:// www.ksbm.oeaw.ac.at/_scripts/php/BR.php?IDtypes=134\&lang=fr>.

Ciceri, Marcella (ed.) (1991): Antón de Montoro, Cancionero, Salamanca, Universidad de Salamanca, Salamanca.

13 Para todo lo anterior es fundamental consultar: Escapa (2000: 29-64) y Garza Merino (2000: 65-95). 
CORDE: REAL ACADEMIA ESPAÑOLA: Banco de datos (CORDE) [en línea]. Corpus diacrónico del español. <http://www.rae.es> [fecha de consulta: 9 de agosto de 2011].

Dutton, Brian (1982): Catálogo/Índice de la poesía cancioneril del siglo XV, Madison, Hispanic Seminary of Medieval Studies.

Escapa, Pablo Andrés, et al. (2000): "El original de imprenta" en Francisco Rico (ed.), Imprenta y crítica textual en el Siglo de Oro, Valladolid, Centro para la Edición de los Clásicos Españoles, pp. 29-64.

Falhauber, Charles B. (1983): Medieval Manuscripts in the Library of the Hispanic Society of America, vol. I, New York, The Hispanic Society of America, pp. 516-18.

Fernández de Villegas, Pedro (1515): La tradución del Dante de lengua toscana en verso castellano, por el Reuerendo don Pedro Fernández de Villegas arcediano de Burgos y por el comentado, allende de los otros glosadores, [...] con otros dos tratados, vno que se dize Querella de la fe y otro Aversión del mundo y conuersión a Dios, Burgos, Fadrique alemán de Basilea. Ejemplar conservado en la Biblioteca del Palacio Real bajo la signatura I-B-21.

Fine, Thomas Rea (1981): Fernández de Villegas's translation and commentary on Dante's "Inferno", University of Michigan, University Microfilms Internarional.

Fosca, Nicola (2003 [2002]): Commento alla "Commedia" di Dante: "Inferno", Hanover, Dartmouth Dante Project.

Garza Merino, Sonia (2000): "La cuenta del original" en Francisco Rico (ed.), Imprenta y crítica textual en el Siglo de Oro, Valladolid, Centro para la Edición de los Clásicos Españoles, pp. 65-95.

Hamlin, Cinthia M. (2011): "El comentario de la Divina Comedia de Villegas y el humanismo peninsular: reflexiones lingüísticas y renovación filológica”, Incipit, XXXI, pp.73-100.

Hamlin, Cinthia M. a (2012a): "Fernández de Villegas y Landino, traducción y reapropiación, el caso de la dicotomía vida activa-vida contemplativa en el Comentario de la Commedia", Ehumanista, 20, pp. 430-450.

Hamlin, Cinthia M. (2012b): "El comentario de la Divina Comedia de Fernández de Villegas: características generales y actitudes humanistas", Ehumanista, 21, pp. 437-466.

Hamlin, Cinthia M. (2012c): "La traducción en la España pre-humanista y sus causas políticoideológicas: el caso de la Divina Commedia y los Reyes Católicos", Revista de Literatura Medieval, 24, pp. 81-100.

Hamlin, Cinthia M. (2012d): "Perspectivas y planteamientos de una poética: reflexiones sobre poesía y ficción en el comentario a la Comedia de Fernández de Villegas”, e-Spania, 14 $<\mathrm{http}: / / \mathrm{e}-$ spania.revues.org/22014>.

Hamlin, Cinthia M. (2012-13): "Reseña de Roberto Mondola, Dante nel Rinascimento castigliano. L'Infierno di Pedro Fernández de Villegas", Incipit, XXXII-XXXIII, pp. 324-333.

Hamlin, Cinthia M. (2013a): "La traducción de la Divina Comedia de Villegas: problemas de datación y filiación de testimonios", Letras, 67-8, pp. 107-116.

Hamlin, Cinthia M. (2013b): "La configuración apologética del comentario de la Divina Comedia (1515): Fernández de Villegas y su reapropiación de las alusiones histórico-míticas del Comento de Landino", Lemir 17, pp. 113-150.

Hamlin, Cinthia M. (2014): "De nuevo sobre la funcionalidad propagandística de la traducción y el comentario de la Divina Comedia de Villegas (1515)”, La Corónica, 42.2 (Spring), en prensa.

Landino, Cristoforo (1481): Divina Commedia di Dante Alighieri: col comento di Christoforo Landino, Firenze, Niccolò di Lorenzo della Magna. Facsímil digital disponible en: <http:// gallica.bnf.fr>.

Maguire, Fiona (ed.) (1995): Cancionero castellano de París [PN5] [BNP Esp. 227] (1430-1470), Madison, Hispanic Seminary of Medieval Studies.

Morreale, Margherita (2006 [1967]): "Apuntes bibliográficos para el tema de 'Dante en España hasta el s. XVII" ", José Luis Rivarola y José Pérez Navarro (eds.), Escritos escogidos de lengua y literatura española, Madrid, Gredos, pp. 213-250. 
Petrocchi, Giorgio (ed.) (1994): Alighieri, Dante, La Divina Commedia secondo l'antica vulgata, Firenze, Le Lettere.

Pianigiani, Ottorino (2004-2008 [1907]): Vocabolario etimologico della lingua italiana. Ed. electrónica de Francesco Bonomi <http://www.etimo.it/>.

Recio, Roxana (1999): "Landino y Villegas: análisis de una traducción del infierno de Dante", Voz y Letra, Revista de Literatura, 10.1, pp. 25-39.

Recio, Roxana (2003): "Comentarios y Lenguas Vernáculas: la traducción como vehículo cultural propagandístico", Revista de Lenguas y literaturas catalana, gallega y vasca, 9, pp. 321-332.

Rico, Francisco (2000): "Crítica textual y transmisión impresa (para la edición de "La Celestina"), en Francisco Rico, Pablo Andreìs, Sonia Garza et al., Imprenta y crítica textual en el Siglo de Oro, Valladolid, Centro para la Edición de los Clásicos Españoles, pp. 223-241.

Rodríguez Velasco, Jesús (2001): "La Bibliotheca y los márgenes: Ensayo teórico sobre la glosa en el ámbito cortesano del siglo XV en Castilla. I: códice, dialéctica y autoridad", eHumanista, 1, pp. 119-134.

Vázquez-Ayora, Gerardo (1977): Introducción a la traductología, Washington, Georgetown University Press.

Fecha de recepción: 17 de noviembre de 2011

Fecha de aceptación: 13 de agosto de 2012 